\title{
Brain Injury in Neonates with Complex Congenital Heart Disease: What Is the Predictive Value of MRI in the Fetal Period?
}

\author{
(D) M. Brossard-Racine, (D)A. du Plessis, (D) G. Vezina, (D). Robertson, (D). Donofrio, (DW. Tworetzky, and (D). Limperopoulos
}

\begin{abstract}
BACKGROUND AND PURPOSE: Brain injury in neonates with congenital heart disease is an important predictor of adverse neurodevelopmental outcome. Impaired brain development in congenital heart disease may have a prenatal origin, but the sensitivity and specificity of fetal brain MR imaging for predicting neonatal brain lesions are currently unknown. We sought to determine the value of conventional fetal MR imaging for predicting abnormal findings on neonatal preoperative MR imaging in neonates with complex congenital heart disease.
\end{abstract}

MATERIALS AND METHODS: MR imaging studies were performed in 103 fetuses with confirmed congenital heart disease (mean gestational age, $31.57 \pm 3.86$ weeks) and were repeated postnatally before cardiac surgery (mean age, $6.8 \pm 12.2$ days). Each MR imaging study was read by a pediatric neuroradiologist.

RESULTS: Brain abnormalities were detected in 17/103 (16\%) fetuses by fetal MR imaging and in 33/103 (32\%) neonates by neonatal MR imaging. Only $9 / 33$ studies with abnormal neonatal findings were preceded by abnormal findings on fetal MR imaging. The sensitivity and specificity of conventional fetal brain MR imaging for predicting neonatal brain abnormalities were $27 \%$ and $89 \%$, respectively.

CONCLUSIONS: Brain abnormalities detected by in utero MR imaging in fetuses with congenital heart disease are associated with higher risk of postnatal preoperative brain injury. However, a substantial proportion of anomalies on postnatal MR imaging were not present on fetal MR imaging; this result is likely due to the limitations of conventional fetal MR imaging and the emergence of new lesions that occurred after the fetal studies. Postnatal brain MR imaging studies are needed to confirm the presence of injury before open heart surgery.

ABBREVIATIONS: $\quad \mathrm{BCH}=$ Boston Children's Hospital; $\mathrm{CHD}=$ congenital heart disease; $\mathrm{CNMC}=$ Children's National Medical Center; $\mathrm{GA}=$ gestational age; $\mathrm{GMH}-\mathrm{IVH}$ = germinal matrix hemorrhage and intraventricular hemorrhage; NHPI = nonhemorrhagic parenchymal injury; $\mathrm{pWMI}=$ punctate white matter injury; SNAP $=$ Scores for Neonatal Acute Physiology

P reoperative brain injury in neonates with complex congenital heart disease (CHD) is prevalent, ranging from $26 \%$ to $41 \%^{1-7}$ and is associated with neurodevelopmental disabilities. ${ }^{8}$ Delayed brain maturation in infants with CHD before

Received September 14, 2015; accepted after revision January 5, 2016

From the Advanced Pediatric Brain Imaging Research Laboratory (M.B.-R., C.L.), Division of Diagnostic Imaging and Radiology (M.B.-R., G.V., C.L.), Fetal and Transitional Medicine (M.B.-R., A.d.P., C.L.), and Division of Cardiology (M.D.), Children's National Health System, Washington DC; and Divisions of Radiology (R.R.) and Cardiology (W.T.), Children's Hospital Boston and Harvard Medical School, Boston, Massachusetts.

This work was supported by the Canadian Institutes of Health Research (MOP81116) and the National Institutes of Health (RO1HL116585, IDDRC, grant P3OHD40677). Dr Marie Brossard-Racine was financially supported by the Canadian Institutes of Health Research at the time of manuscript preparation.

Please address correspondence to Catherine Limperopoulos, PhD, Advanced Pediatric Brain Imaging Research Laboratory, Division of Diagnostic Imaging and Radiology/Fetal and Transitional Medicine, Children's National Medical Center, 111 Michigan Ave NW, Washington DC 20010; e-mail: climpero@childrensnational.org

- Indicates open access to non-subscribers at www.ajnr.org

http://dx.doi.org/10.3174/ajnr.A4716 neonatal surgical repair has also been reported, suggesting an antenatal onset. ${ }^{2,5,9}$

More recently, our findings ${ }^{10}$ are in line with others ${ }^{11}$ who reported that conventional fetal $\mathrm{MR}$ imaging identifies acquired and developmental brain anomalies in up to $39 \%$ of fetuses with CHD. Using quantitative MR imaging, our group has shown that fetuses with CHD demonstrate progressive disturbances in brain growth and delayed cortical gyrification in the third trimester. $^{12,13}$ The extent to which these fetal brain findings are transient or persist in the postnatal preoperative period remains to be determined. Using a prospective longitudinal design, we sought to examine the value of conventional fetal MR imaging for predicting preoperative abnormalities on neonatal MR imaging in neonates with complex CHD. Specifically, we aimed to evaluate the sensitivity and specificity of conventional fetal brain MR imaging as a clinical tool to accurately detect postnatal preoperative brain abnormalities. As a secondary objective, we also examined the relationship between clinical risk factors and newly identified postnatal brain findings. 


\section{MATERIALS AND METHODS Subjects}

In this longitudinal observational study, pregnant women with a fetal diagnosis of complex CHD confirmed by echocardiography were consecutively recruited and prospectively followed. Complex CHD included diagnoses that would require corrective or palliative neonatal open heart surgery. Recruitment took place between February 2007 and December 2010 at Boston Children's Hospital (BCH) and from June 2011 to October 2014 at Children's National Medical Center (CNMC). Subjects with multiple pregnancies, extracardiac anomalies on fetal sonography, congenital infection, documented fetal or neonatal chromosomal anomalies, and/or multiorgan dysmorphic conditions were excluded. Written informed consent was obtained by all study participants. The study was approved by BCH Committee on Clinical Investigation and the institutional review board of CNMC, in compliance with the Health Insurance Portability and Accountability Act.

A fetal echocardiogram was performed by one of the experienced fetal cardiologists at each center. Fetal CHD diagnoses were subsequently categorized as cyanotic versus acyanotic, single- versus 2-ventricle physiology, and with or without aortic obstruction as determined by anatomic classification and prediction of in utero physiology based on the structural defect. Enrolled pregnant women completed fetal MR imaging during the second or the third trimester of pregnancy. Gestational age of the fetuses was estimated from the first-trimester-dating sonography. At the end of 2013, a second fetal MR imaging (ie, 2 fetal MR imaging studies) was added to our study protocol to serially examine fetal brain structure and development during the second and the third trimesters. For this subset $(n=19)$, only the second MR imaging was included in our analyses because the MR imaging results did not differ between the 2 scans. Once medically stable after delivery, neonates underwent preoperative brain MR imaging. Antenatal, perinatal, and postnatal clinical data and demographic information for each neonate was collected through maternal questionnaires and medical record review and were entered into a Research Electronic Data Capture tool (REDCap; http:// www.project-redcap.org/) hosted at CNMC. ${ }^{14}$ Only subjects who completed both fetal and neonatal MR imaging were kept in the analyses.

\section{Fetal MR Imaging Acquisition Protocol}

Each fetal MR imaging was performed on a 1.5T scanner either on an Achieva (Philips Medical Systems, Best, the Netherlands) device by using a 5-channel phased array coil ( $\mathrm{BCH}$ cohort) or on a Discovery scanner (GE Healthcare, Milwaukee, Wisconsin) with an 8-channel phased array coil (CNMC cohort). Two-millimeter multiplanar single-shot fast spin-echo T2-weighted sequences and $4-\mathrm{mm}$ diffusion-weighted images were obtained by using standard clinical parameters. ${ }^{10}$ Diffusion images were assessed at $b=0$ for susceptibility changes and at $b=800$ for diffusion abnormalities; apparent diffusion coefficient maps were also reviewed. Total scan time was approximately 45-60 minutes, and no contrast or sedation was used for any of the fetal MRIs. All studies were reviewed for the presence of developmental malformations, maturation status, and acquired abnormalities by the experienced fetal neuroradiologist at each center following the clinical guidelines in place at the time of the study. Mild ventriculomegaly was diagnosed when the maximum width of the lateral ventricles at the level of the atrium measured between 10 and $15 \mathrm{~mm} .{ }^{15} \mathrm{In}$ creased extra-axial space was diagnosed when the distance between the inner margin of the cranium and the outer margin of the frontal, temporal, parietal, and/or occipital region was considered enlarged for gestational age. ${ }^{16,17}$ Given that the T2 signal of normal periventricular white matter in third-trimester fetuses is often slightly hyperintense compared with the subcortical white matter, T2 signal greater than the background signal of normalappearing white matter was considered abnormal. ${ }^{18}$ Vermian hypoplasia was diagnosed when there was partial absence of the posterior portion of the vermis in the presence of normally shaped cerebellar hemispheres. ${ }^{19}$ Finally, sulcation was considered delayed if $\geq 2$ weeks from the expected published normative data. ${ }^{20}$

\section{Neonatal MR Imaging Acquisition Protocol}

Unless clinically indicated, no sedation or contrast was used during the neonatal MR imaging. The nonsedated neonatal MRIs were performed with the patient under natural sleep after feeding and swaddling. All neonates were immobilized by using an Infant Vacuum Immobilizer (Newmatic Medical, Caledonia, Michigan) and were provided with a double ear protection: ear plugs (Mighty Plugs; Beneficial Products, Ashland, Oregon) and Neonatal Noise Guards (Natus Medical, Seattle, Washington). The neonatal brain MRIs were performed on either a $1.5 \mathrm{~T}$ scanner (Achieva, Philips Healthcare) using a 32-channel receive-only head coil (BCH cohort) or on a 3T Discovery scanner (GE Healthcare) with an 8- or 32-channel receive-only head coil (CNMC cohort). The MR imaging sequences used for clinical diagnosis included 3D-T1WI gradient-echo (fast-field echo) and T2WI dual-echo turbo spin-echo at BCH, 3D-T1WI gradient-echo (echospoiled gradient echo) and 3D-T2WI fast spin-echo (Cube; GE Healthcare) at CNMC, and diffusion-weighted images at both centers. Susceptibility-weighted images were also acquired for the CNMC cohort only because it was not the standard of practice at the time of the MR imaging study at $\mathrm{BCH}$. Total scan time was approximately 45-60 minutes.

All neonatal brain MR imaging studies were reviewed by one of the pediatric neuroradiologists at each center, blinded to the subject's clinical information with the exception of age at the time of the MR imaging. MR imaging findings were classified as developmental or acquired injury. Developmental malformations included ex vacuo ventriculomegaly, increased extra-axial space, and delayed maturation based on gyral and sulcal development. Neonatal vermian hypoplasia was diagnosed when the length of the vermis (ie, superior-inferior extent) was smaller for the expected age in the presence of a normally shaped cerebellar hemisphere. ${ }^{19}$ The corpus callosum was considered hypoplastic when the length was shorter than expected (ie, incomplete development) by using the tectal plate of the midbrain as a reference point for the posterior aspect of the corpus callosum. $^{21}$

Acquired injury was categorized as 1 of the following: 1) punctate white matter injury (pWMI) seen as foci of T1 hyperintensity and/or T2 hypointensity; 2) hemorrhage, parenchymal and non- 
Table 1: CHD diagnoses distribution $(n=103)$

\begin{tabular}{lccc}
\hline \multicolumn{1}{c}{ Diagnosis } & $\begin{array}{c}\text { Distribution } \\
(\%)\end{array}$ & $\begin{array}{c}\text { Abnormal Findings } \\
\text { on Fetal MRI (\%) }\end{array}$ & $\begin{array}{c}\text { Abnormal Findings } \\
\text { on Preop MRI (\%) }\end{array}$ \\
\hline $\begin{array}{l}\text { Dextro-transposition of the } \\
\text { great arteries }\end{array}$ & $31(30.1)$ & $5(29.4)$ & $9(27.3)$ \\
$\begin{array}{l}\text { Hypoplastic left-heart syndrome } \\
\text { Double-outlet right ventricle }\end{array}$ & $20(19.4)$ & $3(17.6)$ & $9(27.3)$ \\
Tetralogy of Fallot & $13(12.6)$ & $2(11.8)$ & $2(6.1)$ \\
Atrioventricular septal defect & $11(10.7)$ & $2(11.8)$ & $2(6.1)$ \\
Tricuspid atresia & $8(7.8)$ & $3(17.6)$ & $1(3.0)$ \\
Ventricular septal defect & $4(3.9)$ & $1(5.9)$ & $3(12.1)$ \\
Pulmonary atresia & $4(3.9)$ & $0(0.0)$ & $2(6.1)$ \\
Ebstein anomaly & $3(2.9)$ & $0(0.0)$ & $2(6.1)$ \\
Pulmonary stenosis & $2(1.9)$ & $0(0.0)$ & $1(3.0)$ \\
Aortic stenosis & $2(1.9)$ & $0(0.0)$ & $1(3.0)$ \\
Severe coarctation of the aorta & $1(1.0)$ & $0(0.0)$ & $0(0.0)$ \\
Hypoplastic right-heart syndrome & $2(1.9)$ & $0(0.0)$ & $0(0.0)$ \\
Truncus arteriosus & $1(1.0)$ & $0(0.0)$ & $0(0.0)$ \\
Total & $1(1.0)$ & $1(5.9)$ & $1(3.0)$ \\
\hline
\end{tabular}

Note:--Preop indicates preoperative.

parenchymal (excluding birth-related subarachnoid and subdural hemorrhages); and 3) nonhemorrhagic parenchymal injury (NHPI). To differentiate mild pWMI from moderate/severe pWMI, we used a cutoff of $\geq 3$ lesions and $<2 \mathrm{~mm}$ as commonly used in imaging studies of at-risk neonates. ${ }^{6,22}$ We could confirm the hemorrhagic nature of the punctate lesions when susceptibilityweighted images were acquired (ie, CNMC cohort) and corresponding hypointense foci on the $\mathrm{T} 2{ }^{\star}$-weighted images were detected. A larger parenchymal hemorrhage was considered mild when the intracerebral hemorrhage volume corresponded to $<2 \%$ of the total brain volume. ${ }^{23}$

Nonparenchymal hemorrhages included choroid plexus hemorrhage, germinal matrix hemorrhage, and intraventricular hemorrhage (GMH-IVH) graded according to the Papile classification. ${ }^{24}$ NHPIs were further classified as the following: 1) focal infarction of various etiologies (eg, vaso-occlusive, embolic, hypoperfusion) that appeared as hyperintense on T2weighted images and/or a hyperintense focal area on diffusion images; 2) diffuse injury (ie, diffuse excessive high signal intensity when the T2 signal exceeded the signal of the corresponding normal-appearing white matter); and 3) cysts.

\section{Statistical Analysis}

Descriptive statistics using means, SDs, and frequencies were used to characterize the sample and the nature of the brain abnormalities described by conventional MR imaging. The relative risks with $95 \%$ confidence intervals were calculated to assess the association between abnormal findings on ante- and postnatal brain MR imaging studies. The sensitivity and specificity of fetal brain MR imaging were then calculated. Positive and negative predictive values were also calculated to characterize the accuracy of fetal brain MR imaging. Maternal and subject characteristics and clinical factors in newly injured-versus-noninjured neonates were compared by using an independent samples $t$ test for continuous parametric variables, the Mann-Whitney $U$ test for continuous nonparametric and ordinal variables, and the Fisher exact test for categoric variables. All analyses were executed by using SPSS, Version 17 (IBM, Armonk, New York).

\section{RESULTS}

\section{Sample Characteristics}

A total of 150 pregnant women completed fetal MR imaging as part of our prospective study. As described in our "Materials and Methods" section, a subset $(n=19)$ of women completed 2 fetal MR imaging studies, resulting in 169 MR images obtained at a mean gestational age (GA) of $31.57 \pm 3.86$ weeks (range, 17.71-38.57 weeks). Of the 150 subjects with CHD, 2 died in utero and 9 died in the early postnatal period before MR imaging could be performed. Twelve families declined the neonatal MR imaging. Eight families had moved out of state or had changed contact information and could not be located. Lastly, 16 neonates did not undergo a neonatal MR imaging because they were deemed too unstable medically by the treating team. Subject characteristics of the subset of neonates did not significantly differ from the rest of the sample with respect to sex, CHD diagnosis, frequency of fetal MR imaging abnormality, birth weight, gestational age at birth, Apgar score, and Scores for Neonatal Acute Physiology (SNAP) score. However, neonates that completed the preoperative MR imaging were more likely to be born via vaginal delivery $(P=.01)$.

The final sample consisted of 103 fetuses (61 males) with CHD who completed a fetal MR imaging at a mean GA of $32.71 \pm 3.61$ weeks (median, 33.71 weeks; range, 23.86-38.57 weeks) and then a neonatal MR imaging study at mean age of $6.8 \pm 12.2$ days (median, 3 days; range, 0-76 days). Of note, all except 5 infants were scanned before 26 days of life. The neonatal MR imaging was performed, on average, 6.8 weeks (range, 1-21 weeks) after the last fetal MR imaging. The sample included 48 neonates from the Boston cohort and 55 from CNMC. The 4 most common cardiac diagnoses in our sample were transposition of the great arteries (30\%), hypoplastic left-heart syndrome (19\%), double-outlet right ventricle (13\%), and tetralogy of Fallot (11\%). The complete distribution of the CHD diagnoses is presented in Table 1. The frequency of abnormal MR imaging studies for both fetal and postnatal MR imaging for each CHD diagnosis is also reported in Table 1. Subsequent classification by cardiac diagnosis of our sample resulted in 74 cyanotic versus 29 acyanotic physiology; 46 with single-ventricle versus 57 with 2-ventricle physiology, and 33 with aortic obstruction versus 70 without.

The $\mathrm{BCH}$ and $\mathrm{CNMC}$ cohorts did not differ with respect to the maternal and neonatal subjects' characteristics, except for gestational diabetes, which was present in 7 cases ( 4 on medication and 3 diet-controlled), all from the CNMC cohort $(P=.014)$. A higher proportion of neonates with $\mathrm{CHD}$ were intubated at birth in the CNMC cohort (16 versus 3; $P<.001$ ), while a significantly higher percent of neonates from the Boston cohort underwent a cardiac catheterization before neonatal MR imaging ( 9 versus $2 ; P=.012$ ). No other differences in clinical practice were found between the 2 centers. The antenatal, in- 
trapartum, and perinatal characteristics of the sample are presented in Table 2.

\section{Brain Abnormalities Detected by Conventional Brain MR Imaging}

On conventional fetal MR imaging, brain abnormalities were detected in 17 cases $(16.3 \%)$ and included the following: isolated mild ventriculomegaly (6/17) (Fig $1 A$ ), increased extra-axial spaces (4/17) (Fig 1B), white matter cysts (2/17), isolated inferior vermian hypoplasia (2/17), and white matter signal hyperintensity on T2-weighted images (1/17). Of note, one of the fetuses with ventriculomegaly also had a single frontal subependymal cyst. Two fetuses presented with an immature brain appearance based on sulcal-gyral development.

Thirty-two neonates (32.0\%) had abnormal findings on conventional preoperative brain MR imaging. Acquired injury was

\section{Table 2: Clinical characteristics of the cohort $(n=103)$}

\begin{tabular}{lc}
\hline \multicolumn{1}{c}{ Antenatal } & $\begin{array}{c}\text { Mean } \pm \text { SD/Median } \\
\text { (Range)/No. (\%) }\end{array}$ \\
\hline Pregnancy-induced diabetes & $7(6.8 \%)$ \\
Group B streptococcus & $13(13.3 \%)$ \\
Hyperthyroidism & $3(2.9 \%)$ \\
Hypothyroidism & $8(7.8 \%)$ \\
Pregnancy-induced hypertension & $3(2.9 \%)$ \\
Placenta previa & $3(2.9 \%)$ \\
Placenta abruption & $2(1.9 \%)$ \\
Preterm labor & $3(2.9 \%)$ \\
Chorioamnionitis & $1(1.0 \%)$ \\
Intrapartum & \\
Induced labor & $48(52.7 \%)$ \\
Emergency cesarean delivery & $22(21.8 \%)$ \\
Gestational age at birth (wk) & $38.5 \pm 1.3$ \\
Birth weight (g) & $3173 \pm 552$ \\
Apgar score 1 min/5 min & $8(1-9) / 8(5-9)$ \\
Intubation at birth & $18(19 \%)$ \\
SNAP-II score & $5(0-68)$ \\
Perinatal (prior to MRI) & \\
Lowest pH & $7.318 \pm 0.062$ \\
Lowest pO ${ }_{2}$ & $38.63 \pm 23.72$ \\
Highest pCO & $47.65 \pm 12.00$ \\
Cardiac catheterization & $11(10.9 \%)$ \\
Balloon atrial septostomy & $15(14.6 \%)$ \\
\hline
\end{tabular}

Note:- $\mathrm{pCO}_{2}$ indicates carbon dioxide partial pressure; $\mathrm{pO}_{2}$, oxygen partial pressure.
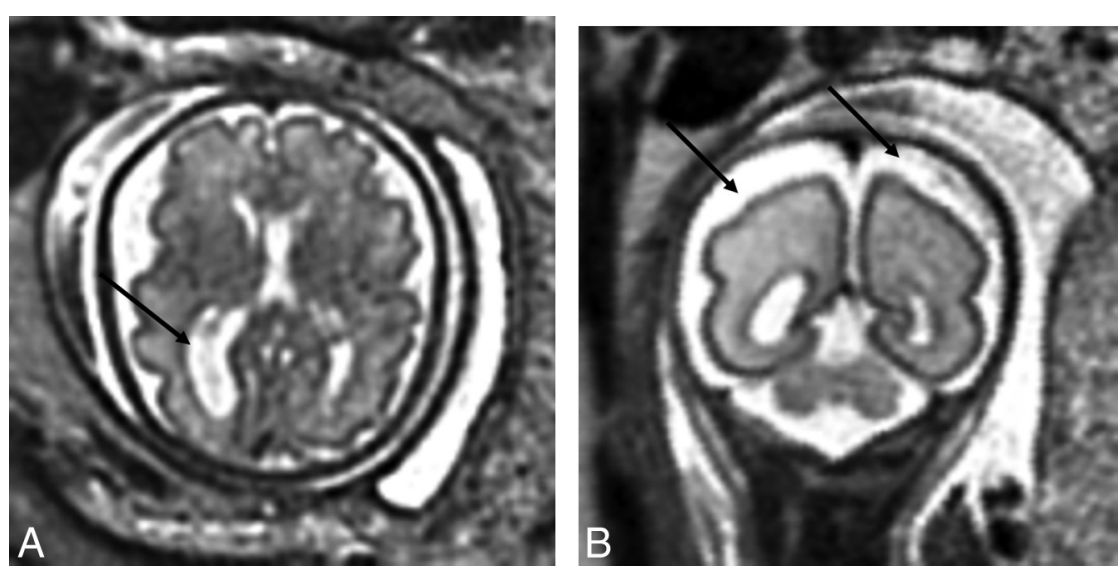

FIG 1. A, T2-weighted axial view of the brain of a 32-week-old fetus with CHD with unilateral ventriculomegaly. $B, T 2$-weighted axial view of the brain of a 27.29-week-old fetus with CHD with extra-axial spaces. Reprinted from Brossard-Racine et al. ${ }^{10}$ present in $27(26.2 \%)$ neonates. An isolated developmental anomaly (ie, without acquired injury) was detected in 5 neonates, while 5 others presented with both developmental and acquired findings. Hemorrhagic and nonhemorrhagic pWMIs were present in 15 neonates ( 5 mild, 10 moderate/severe), most commonly observed in the periventricular white matter, the centrum semiovale (Fig 2), and the frontal white matter. NHPI was the second most frequently acquired injury and was detected in 13 neonates. Of these, 7 presented with diffuse abnormal T2 signal prolongation (isolated in 2) in the periventricular white matter and 3 with focal infarctions in the deep gray matter (isolated in 1) (Fig 3). Three neonates presented with cysts: One had an isolated right germinal matrix cyst, 1 had a single cyst in the periventricular white matter, and 1 had multiple cysts in the bilateral frontal white matter.

Nonparenchymal hemorrhages were reported in 11 neonates (isolated in 3 cases) and included the following: intraventricular hemorrhage in 1, GMH-IVH grade-I in 1, GMH-IVH grade-II in 5 , GMH-IVH grade-III in 1, and choroid plexus hemorrhage in 3. Mild parenchymal hemorrhages were observed in 2 neonates, 1 in the cerebellum (Fig 4) and the other in the left globus pallidus.

Developmental brain malformations were described in 10 (9.7\%) neonates. Three neonates presented with isolated congenital malformation such as mild vermis hypoplasia (1/3) and hypoplasia of the corpus callosum (2/3). Delayed maturation was reported in 1 neonate, which manifested as delayed opercular closure. The other 6 neonates presented with a mild global atrophy of the cerebral white matter (isolated in 2 cases), qualitatively described by a mild degree of ventricular enlargement and/or extra-axial space in the intracranial cavity.

\section{Sensitivity and Specificity of Conventional Fetal Brain MR Imaging}

The relative risk of presenting with abnormal findings on postnatal brain MR imaging after abnormal findings on fetal brain MR imaging was $1.90(95 \% \mathrm{CI}, 1.08-3.33 ; P=.03)$. This translated into a sensitivity of $27.3 \%$ and a specificity of $88.6 \%$ (Table 3 ). The positive and the negative predictive values of conventional fetal MR imaging for identifying persisting neonatal brain abnormality were $52.9 \%$ and $72.1 \%$, respectively.

The distribution of prenatal brain abnormalities and how they manifested postnatally is presented in Table 4. Of the 17 fetuses with abnormal findings on prenatal brain MR imaging, 9 also presented with abnormal findings on neonatal MR imaging, while 8 had normal findings on a neonatal study. The fetal brain abnormalities that resolved or normalized by the time of the neonatal MR imaging included mild extracerebral space (2/8), mild unilateral ventriculomegaly (3/8), immature brain appearance (2/8), vermis hypoplasia (1/8), and single frontal subependymal cyst (1/ 8). Gestational age at fetal MR imaging of the fetuses who had transient fetal 


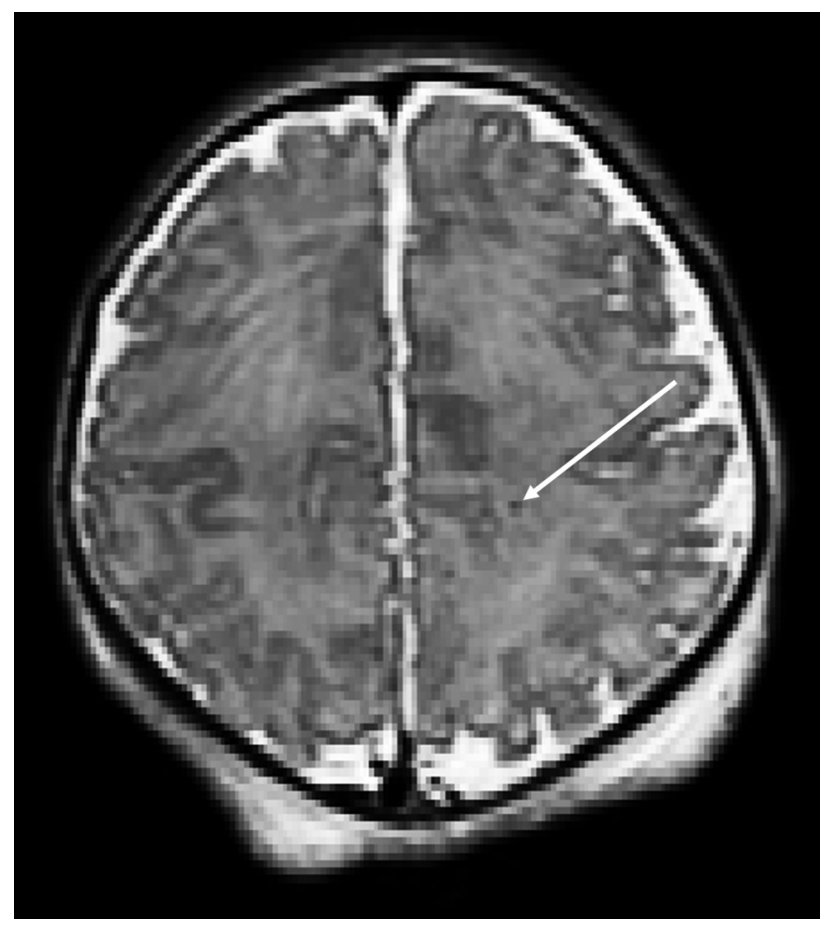

FIG 2. T2-weighted axial view of a neonate with $\mathrm{CHD}$ with punctate white matter injury.

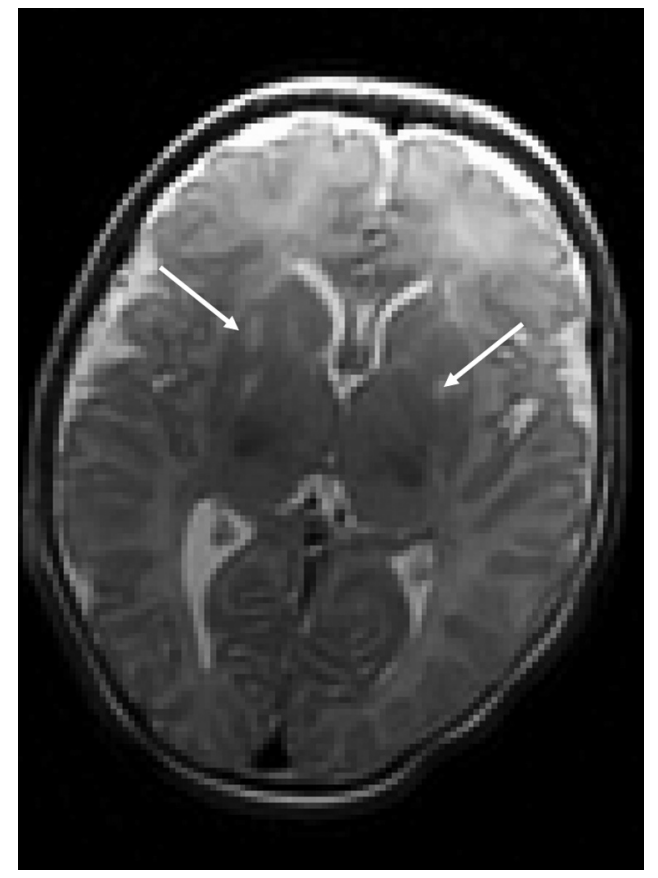

FIG 3. T2-weighted axial view of a neonate with $\mathrm{CHD}$ with bilateral deep gray matter infarcts.

brain findings was not significantly different from those fetuses without abnormalities or those with findings on both fetal and neonatal MR imaging. However, fetuses with abnormal fetal MR imaging findings and cyanotic CHD were significantly more likely to present with brain abnormalities on neonatal MR imaging ( 8 versus $1, P=.05$ ). This association was not found when comparing single-ventricle and 2-ventricle physiology or aortic obstruction.

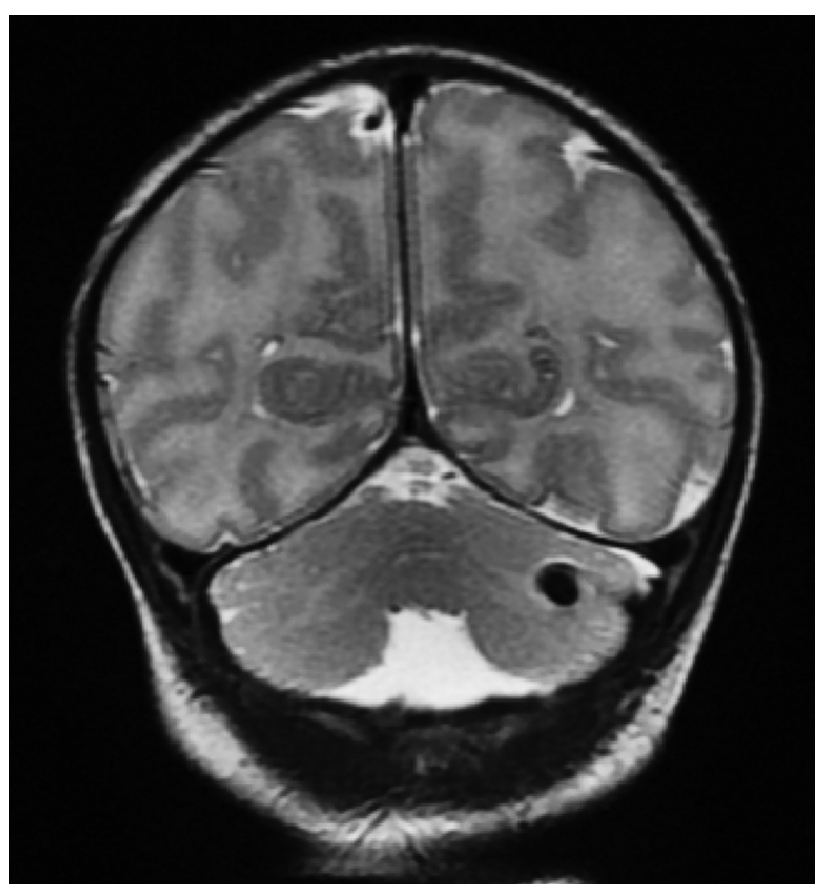

FIG 4. T2-weighted coronal view of a neonate with $\mathrm{CHD}$ with unilateral cerebellar hemorrhage.

Of the 9 cases with CHD that presented with brain abnormalities on both the fetal and neonatal scans, 5 neonatal findings were already seen on the fetal MR imaging. These included 2 neonates with white matter cysts, 1 of the 2 neonates with vermis hypoplasia and 1 with sustained mild ventricular enlargement. Of note, the 2 fetal cases that had persisting white matter cysts also presented with other parenchymal injury on the neonatal MR imaging. In 4 other cases, abnormalities were reported on both the fetal and neonatal brain MR imaging, but the nature of the findings had evolved between fetal and neonatal scans. These included 2 cases of mild extra-axial space on the fetal MR imaging that then presented with moderate/severe pWMI on neonatal MR imaging: 1 fetus with an additional choroid plexus hemorrhage and 1 fetus with mild ventriculomegaly presenting with solely diffuse NHPI on the neonatal MR imaging. Last, the fetus with isolated increased T2 signal presented with a grade II intraventricular hemorrhage on neonatal MR imaging.

\section{New Postnatal Brain MR Imaging Findings}

New brain abnormalities on neonatal MR imaging were detected in $24(27.9 \%)$ neonates who had previous normal findings on fetal MR imaging. These included 20 neonates with newly acquired injuries, 4 of whom also presented with cerebral atrophy. New isolated developmental anomalies were detected in 4 neonates and included 2 cases with hypoplasia of the corpus callosum, delayed opercular closure in one and isolated global cerebral atrophy in another.

Newly acquired brain injury identified by neonatal MR imaging (ie, detected after a normal findings on fetal MR imaging) included 13 of the 15 postnatal cases with pWMI, 10 of the 11 postnatal cases with nonparenchymal hemorrhages, 8 of the 14 postnatal cases with neonatal NHPI, and 1 case with mild cerebellar parenchymal hemorrhage. These new NHPIs presented as fo- 
cal infarctions in 2 neonates, diffuse injuries in 5 neonates, and isolated germinal matrix cyst in 1 .

\section{Determinants of Newly Acquired Postnatal Brain MR Imaging Abnormalities}

Neonates with newly detected brain findings were not significantly different from neonates with normal findings on postnatal brain MR imaging with respect to their ante- and perinatal characteristics. However, although there was no significant difference in GA at birth or in days of life at neonatal MR imaging, the 20 neonates with newly acquired brain MR imaging findings were imaged postnatally at a significantly younger GA than the noninjured neonates $(\mathrm{GA}=38.74$ versus 39.73 weeks; $t=2.49, P=$ $.015)$ and had their neonatal MR imaging performed earlier following the fetal MR imaging (5.11 versus 7.08 weeks; $P=.011$ ). Newly acquired brain lesions were significantly more frequent in neonates with single-ventricle CHD ( 15 versus $5 ; P=.002$ ) and in those who underwent cardiac catheterization before the MR im-

Table 3: Fetal MRI sensitivity, specificity, and positive and negative predictive value

\begin{tabular}{lcccc}
\hline \multirow{2}{*}{$\begin{array}{c}\text { Abnormal Fetal } \\
\text { MRI Findings }\end{array}$} & \multicolumn{3}{c}{$\begin{array}{c}\text { Abnormal Neonatal } \\
\text { MRI Findings }\end{array}$} & \\
\cline { 2 - 4 } Yes & 9 & No & Total & Predictive Value \\
No & 24 & 8 & 17 & $\begin{array}{c}\text { Positive, } \\
9 / 17=52.9 \% \\
\text { Total }\end{array}$ \\
& 33 & 62 & 86 & $\begin{array}{c}\text { Negative, } \\
62 / 86=72.1 \%\end{array}$ \\
& $\begin{array}{c}\text { Sensitivity, } \\
9 / 33=27.3 \%\end{array}$ & $\begin{array}{c}\text { Specificity, } \\
62 / 70=88.6 \%\end{array}$ & $n=103$ & \\
\hline
\end{tabular}

Table 4: Fetal and neonatal preoperative brain MRI findings ${ }^{a}$

\begin{tabular}{lrccccc}
\hline & Normal & pWMI & Hemorrhage & NHPI & Dev. Abn. ${ }^{\text {b }}$ & Total $^{\text {Normal }}$ \\
\hline N2 & 11 & 6 & 3 & 4 & 86 \\
Extracerebral space & 2 & 2 & 0 & 0 & 0 & 4 \\
Ventriculomegaly & 3 & 0 & 0 & 2 & 1 & 6 \\
WM abn. & 1 & 0 & 1 & 1 & 0 & 3 \\
Immature brain & 2 & 0 & 0 & 0 & 0 & 2 \\
Vermis hypoplasia & 0 & 1 & 0 & 0 & 1 & 2 \\
Total & 70 & 14 & 7 & 6 & 6 & 103 \\
\hline
\end{tabular}

Note:-Dev. Abn. indicates developmental abnormalities.

${ }^{a}$ Cases with multiple abnormalities were included only once in the table on the basis of the most severe finding.

${ }^{b}$ Developmental anomaly includes congenital malformation, delayed maturation, and cerebral atrophy (extra-axial space and/or ventricular enlargement) aging $(P=.006)$. Most interesting, neonates with newly detected lesions were more frequently born by vaginal delivery than those without new lesions, but the association was not significant $(P=$ .082). No other patient-specific or clinical characteristics were associated with newly diagnosed brain injuries. Group differences are presented in Table 5.

\section{DISCUSSION}

This study reports, for the first time, the relationship between serial prenatal and postnatal preoperative conventional brain MR imaging studies in a large cohort of fetuses diagnosed with complex CHD. Brain abnormalities were detected in $17 \%$ prenatally and in almost twice as many (32\%) postnatally before open heart surgery. Noteworthy, CHD cases with abnormal conventional fetal MR imaging findings had a $90 \%$ increased risk of presenting with abnormal neonatal brain MR imaging findings. Although conventional fetal MR imaging was shown to have good specificity, it lacked sensitivity for predicting postnatal brain abnormalities.

As previously reported, the most common fetal abnormalities detected on conventional fetal MR imaging in our cohort consisted of enlargement of the ventricular and extra-axial CSF spaces, which are thought to be markers of delayed brain development in fetuses with CHD. ${ }^{10}$ These qualitative fetal brain findings are consistent with quantitative MR imaging studies, which have reported cerebral parenchymal growth disturbances, increased CSF volume, ${ }^{13}$ and delayed cortical maturation ${ }^{12}$ in fetuses with CHD. However, one of the new findings in the current study is that almost half (8/17) of the fetal brain abnormalities were transient and no longer seen on conventional neonatal MR imaging performed an average of 7 weeks after the fetal study. To our knowledge, there are very few studies that have examined the accuracy of fetal brain MR

Table 5: Clinical characteristics of neonates with CHD with and without newly acquired brain injury ${ }^{a}$

\begin{tabular}{lccc}
\hline & $\begin{array}{c}\text { Neonates without Injury } \\
(\boldsymbol{n}=62)\end{array}$ & $\begin{array}{c}\text { Neonates with New Injury } \\
(\boldsymbol{n}=\mathbf{2 0})\end{array}$ & \multicolumn{1}{c}{$\boldsymbol{P}$ Value } \\
\hline Induced labor & $28(45.2 \%)$ & $10(50.0 \%)$ & .570 \\
Vaginal delivery & $29(46.8 \%)$ & $15(65.0 \%)$ & .082 \\
Gestational age at birth (wk) & $38.93(33.86-40.71)$ & $38.71(36.00-40.00)$ & .134 \\
Birth weight (g) & $3300(1077-4276)$ & $3085(2240-4220)$ & .525 \\
Apgar score 1 min/5 min & $8(1-9) / 8(5-9)$ & $8(5-9) / 8(7-9)$ & $.816 / .266$ \\
Intubation at birth & $14(22.5 \%)$ & $3(12.5 \%)$ & .373 \\
SNAP-II score & $5(0-43)$ & $5(0-28)$ & .471 \\
Lowest pH prior to MRI, (mean) & $7.320(7.174 \pm 7.490)$ & $7.326(7.220 \pm 7.454)$ & .567 \\
Lowest pO 2 prior to MRI & $34.85(20-214)$ & $33.75(22-78)$ & .673 \\
Highest pCO 2 prior to MRI & $46.50(22-83)$ & $46.25(24-61)$ & .794 \\
Cardiac catheterization prior to MRI & $3(4.8 \%)$ & $6(30.0 \%)$ & .006 \\
Balloon atrial sepstostomy prior to MRI & $10(16.1 \%)$ & $4(20.0 \%)$ & .612 \\
Age at MRI (days) & $3.0(1.0-76.0)$ & $3.0(1.0-12.0)$ & .063 \\
Time between fetal and neonatal MRI (wk) & $6.00(1.28-20.85)$ & $4.86(1.00-10.43)$ & .011 \\
\hline
\end{tabular}

${ }^{a}$ Values are median (range) or No. (\%). 
imaging by comparing findings with postnatal imaging. Although fetal MR imaging is thought to be reliable in detecting congenital malformations of the central nervous system, ${ }^{25,26}$ available evidence suggests that some of the variability is attributable to differences in defining normal variants. Isolated mild unilateral ventriculomegaly, for example, has been shown to normalize after birth in $40 \%$ of clinical referrals. ${ }^{27,28}$ Similarly, prenatal isolated mild vermis hypoplasia has been shown to normalize in $32 \%$ by the time of postnatal MR imaging evaluation. ${ }^{29}$ Together these findings call for extra caution when interpreting mild prenatal brain findings of unknown etiology on conventional MR imaging because a high proportion of these subtle markers of delayed brain development may normalize by the end of the pregnancy.

The $26 \%$ prevalence of preoperatively acquired injury in our cohort with a predominance of pWMI is in agreement with that in previous reports. ${ }^{2,5,9,30,31}$ An important finding of this study is that more than two-thirds (24/33) of the postnatal brain abnormalities identified by conventional MR imaging were diagnosed following a fetal MR imaging study with normal findings. The presence of pWMI, hemorrhages, and ischemic infarctions on neonatal MR imaging were new findings that were not seen on the fetal MR imaging studies. This discrepancy between the fetal-versus-postnatal MR imaging studies may be explained, in part, by the current limitations of fetal MR imaging (ie, fetal and maternal motion, image resolution) and the limited acquisition protocol repertoire available for fetal imaging (ie, lack of robust T1weighted and susceptibility-weighted sequences), which might have diminished our ability to detect acute white matter injury in utero. $^{32,33}$ Moreover, it is possible that the third-trimester fetal MR imaging studies were performed before these injuries had occurred or consolidated or were below the threshold for lesion detection by conventional MR imaging.

Delayed brain growth and maturation have been reported postnatally in neonates with hypoplastic left-heart syndrome and dextro-transposition of the great arteries ${ }^{9}$ and have been found to be associated with a greater likelihood of brain injury on preoperative brain MR imaging in neonates with CHD. ${ }^{1,2,4}$ Our results corroborate the finding that delayed brain growth and brain immaturity already evident prenatally are associated with an increased vulnerability to acquired injury. In our cohort, more than one-third (10/27) of the acquired neonatal brain injuries were seen concomitantly with qualitative markers of immaturity either on fetal MR imaging or neonatal MR imaging. Our prospectively collected longitudinal data of ante- and postnatal imaging suggest that these newly acquired injuries may result from the cumulative effect of brain dysmaturity, with hemodynamic insults occurring in the perinatal period or during the complex and potentially hazardous transition from fetal to postnatal circulation. ${ }^{34}$ In fact, we found that the cases with abnormal fetal MR imaging findings (ie, fetal immaturity) and a cyanotic cardiac defect (ie, hemodynamic) were significantly more likely to present with brain abnormalities on neonatal MR imaging. Although the current data did not highlight a single birth-related risk factor for brain lesions, the borderline significant association found between new injury and vaginal delivery raises the question of optimal delivery management for fetuses with complex CHD. Future research is needed to address this important question.
Our data show that preoperative brain injury was significantly more frequent in neonates who underwent a cardiac catheterization before their preoperative MR imaging study. Neonates that required cardiac catheterization in our study all had cyanotic lesions, and most (7/9) had single-ventricle CHD, indicating greater CHD and illness severity among this subset of neonates. Previous studies have reported an association between balloon atrial septostomy and preoperative stroke in neonates with transposition of the great arteries. ${ }^{3,4,6}$ Taken together, these data suggest that cardiac procedures in neonates with CHD with significant hypoxemia and hemodynamic instability are more likely to be associated with brain injury before surgery.

Most interesting, neonates with newly acquired injury had their neonatal MR imaging at a significantly earlier gestational age and sooner after the fetal MR imaging than the noninjured ones. However, we were unable to identify any clinical factors that could explain why these children were scanned earlier. The finding that acquired injury was documented less frequently in neonates scanned at a later time may be explained, in part, by potential resolution of the more subtle injury (eg, pWMI) in the weeks after birth and by the limited time window when diffusion imaging reveals acute/cytotoxic cerebral injury due to subsequent pseudonormalization. ${ }^{35}$ Additionally, we might have missed microhemorrhages in the $\mathrm{BCH}$ cohort, given that susceptibilityweighted imaging was not a standard of practice at the time of the data collection.

Moreover, and in line with other reports, we did not find significant associations between brain injury and other perinatal clinical factors such as birth asphyxia or other markers of illness severity. ${ }^{1,2,5,7,31}$ Nevertheless, earlier age at MR imaging may reflect greater hemodynamic compromise that has not been captured in the current study. Postoperative follow-up MR imaging studies are currently underway and will, hopefully, provide additional insight into this initial observation.

Although this study includes the largest sample reporting serial imaging performed ante- and postnatally, our study limitations deserve mention. First, our current sample size may have been underpowered to identify less frequent clinical risk factors for brain injury. Moreover, given that we carefully excluded cases with chromosomal or genetic conditions, as well as dysmorphic features a priori, it is possible that the true prevalence of brain abnormalities in the overall CHD population has been underestimated in the current sample because structural abnormalities are more frequent in neonates with $\mathrm{CHD}$ and other congenital malformations. ${ }^{36,37}$ Also, approximately $11 \%$ of our sample was deemed by the clinical team as not medically stable enough to undergo a preoperative MR imaging. We may have missed brain injury in this subset of more severely ill neonates.

Nevertheless, this is the first study to provide prospective serial fetal and preoperative neonatal MR imaging data in subjects with complex CHD. Our large CHD sample was not confined to the most severe cardiac diagnoses but rather included a representative range of CHD. Second, although we did not find a significant difference in GA at fetal MR imaging between fetuses with CHD with and without brain abnormalities, it is possible that the timing of our fetal MR imaging limited our ability to detect delayed brain development. For example, it has been reported that GA estima- 
tion based on cortical gyrification can only be made accurately after 28 weeks. ${ }^{38}$ Although only 7 fetuses were scanned during the late second trimester (ie, 24-27 weeks), we did not identify any structural brain abnormalities in fetuses younger than 32 weeks in our cohort. However, our evaluation of the fetal brain was carefully made and took in account a number of gestational-age-appropriate developmental landmarks. Nevertheless, more studies are needed to determine how GA influences the predictive value of fetal MR imaging postnatally. Finally, our overall goal was to evaluate the sensitivity and specificity of conventional fetal MR imaging for predicting postnatal brain abnormalities in neonates with complex CHD before open heart surgery. Another limitation is that we did not perform repeat blinded MR imaging interpretations to assess intra- and interreader reliability. However, our study was conducted in 2 major referral centers for complex neonatal CHD repair, and all MR imaging studies were reviewed by an experienced pediatric neuroradiologist following clinical best practice standards. Consequently, we are confident that the findings reported in this study are representative of the current findings in clinical settings.

\section{CONCLUSIONS}

This study reports, for the first time, that abnormal findings on conventional fetal brain MR imaging are associated with an increased incidence of brain abnormalities in neonates with complex CHD before open heart surgery. Although conventional fetal brain MR imaging had high specificity, it lacked sensitivity in predicting postnatal brain abnormalities. In addition to prenatal brain immaturity, our findings suggest that other currently unidentified clinical and birth-related factors may predispose these neonates to the hazards of cerebral hypoxemia, hypoperfusion, and hemodynamic instability that can occur during the demanding third trimester and the complex circulatory transition surrounding birth.

\section{ACKNOWLEDGMENTS}

We thank the families who participated in the study as well as Lauren Crowder and Samantha Bauer for their assistance in the management of the clinical data.

Disclosures: Marie Brossard-Racine—RELATED: Other: postdoctoral fellowship support from Canadian Institutes of Health Research. Adre du Plessis-RELATED: Grant: National Heart, Lung, and Blood Institute, ${ }^{*}$ Comments: I am coinvestigator on this grant. Catherine Limperopoulos—RELATED: Grant: Canadian Institutes of Health,* National Institutes of Health.* *Money paid to the institution.

\section{REFERENCES}

1. Andropoulos DB, Hunter JV, Nelson DP, et al. Brain immaturity is associated with brain injury before and after neonatal cardiac surgery with high-flow bypass and cerebral oxygenation monitoring. J Thorac Cardiovasc Surg 2010;139:543-56 CrossRef Medline

2. Beca J, Gunn J, Coleman L, et al. New white matter brain injury after infant heart surgery is associated with diagnostic group and the use of circulatory arrest. Circulation 2013;127:971-79 CrossRef Medline

3. Block AJ, McQuillen PS, Chau V, et al. Clinically silent preoperative brain injuries do not worsen with surgery in neonates with congenital heart disease. J Thorac Cardiovas Surg 2010;140:550 -57 CrossRef Medline

4. Dimitropoulos A, McQuillen PS, Sethi V, et al. Brain injury and development in newborns with critical congenital heart disease. Neurology 2013;81:241-48 CrossRef Medline

5. Mahle WT, Tavani F, Zimmerman RA, et al. An MRI study of neurological injury before and after congenital heart surgery. Circulation 2002;106(12 suppl 1):I109-14 Medline

6. McQuillen PS, Hamrick SE, Perez MJ, et al. Balloon atrial septostomy is associated with preoperative stroke in neonates with transposition of the great arteries. Circulation 2006;113:280-85 CrossRef Medline

7. Miller SP, McQuillen PS, Hamrick S, et al. Abnormal brain development in newborns with congenital heart disease. N Engl J Med 2007; 357:1928-38 CrossRef Medline

8. Gaynor JW, Stopp C, Wypij D, et al; International Cardiac Collaborative on Neurodevelopment (ICCON) Investigators. Neurodevelopmental outcomes after cardiac surgery in infancy. Pediatrics 2015; 135:816-25 CrossRef Medline

9. Licht DJ, Shera DM, Clancy RR, et al. Brain maturation is delayed in infants with complex congenital heart defects. J Thorac Cardiovasc Surg 2009;137:529-36; discussion 536-37 CrossRef Medline

10. Brossard-Racine M, du Plessis AJ, Vezina G, et al. Prevalence and spectrum of in utero structural brain abnormalities in fetuses with complex congenital heart disease. AJNR Am J Neuroradiol 2014;35: 1593-99 CrossRef Medline

11. Mlczoch E, Brugger P, Ulm B, et al. Structural congenital brain disease in congenital heart disease: results from a fetal MRI program. Eur J Paediatr Neurol 2013;17:153-60 CrossRef Medline

12. Clouchoux C, du Plessis AJ, Bouyssi-Kobar M, et al. Delayed cortical development in fetuses with complex congenital heart disease. Cereb Cortex 2013;23:2932-43 CrossRef Medline

13. Limperopoulos C, Tworetzky W, McElhinney DB, et al. Brain volume and metabolism in fetuses with congenital heart disease: evaluation with quantitative magnetic resonance imaging and spectroscopy. Circulation 2010;121:26-33 CrossRef Medline

14. Harris PA, Taylor R, Thielke R, et al. Research electronic data capture (REDCap): a metadata-driven methodology and workflow process for providing translational research informatics support. J Biomed Inform 2009;42:377-81 CrossRef Medline

15. Wax JR, Bookman L, Cartin A, et al. Mild fetal cerebral ventriculomegaly: diagnosis, clinical associations, and outcomes. Obstet Gynecol Surv 2003;58:407-14 Medline

16. Girard NJ, Raybaud CA. Ventriculomegaly and pericerebral CSF collection in the fetus: early stage of benign external hydrocephalus? Childs Nerv Syst 2001;17:239-45 CrossRef Medline

17. Watanabe Y, Abe S, Takagi K, et al. Evolution of subarachnoid space in normal fetuses using magnetic resonance imaging. Prenat Diagn 2005;25:1217-22 CrossRef Medline

18. Raybaud C, Ahmad T, Rastegar N, et al. The premature brain: developmental and lesional anatomy. Neuroradiology 2013;55(suppl 2): 23-40 CrossRef Medline

19. Tarui T, Limperopoulos C, Sullivan NR, et al. Long-term developmental outcome of children with a fetal diagnosis of isolated inferior vermian hypoplasia. Arch Dis Child Fetal Neonatal Ed 2014;99: F54-58 CrossRef Medline

20. Garel C. MRI of the Fetal Brain: Normal Development and Cerebral Pathologies. Berlin: Springer; 2004

21. Paul LK. Developmental malformation of the corpus callosum: a review of typical callosal development and examples of developmental disorders with callosal involvement. J Neurodev Disord 2011; 3:3-27 CrossRef Medline

22. Miller SP, Cozzio CC, Goldstein RB, et al. Comparing the diagnosis of white matter injury in premature newborns with serial MR imaging and transfontanel ultrasonography findings. AJNR Am JNeuroradiol 2003;24:1661-69 Medline

23. Beslow LA, Ichord RN, Gindville MC, et al. Pediatric intracerebral hemorrhage score: a simple grading scale for intracerebral hemorrhage in children. Stroke 2014;45:66-70 CrossRef Medline

24. Barkovich AJ. Pediatric Neuroimaging. 4th ed. Philadelphia: Williams \& Wilkins; 2005 
25. Blaicher W, Bernaschek G, Deutinger J, et al. Fetal and early postnatal magnetic resonance imaging: is there a difference? J Perinat Med 2004;32:53-57 Medline

26. Pugash D, Brugger PC, Bettelheim D, et al. Prenatal ultrasound and fetal MRI: the comparative value of each modality in prenatal diagnosis. Eur J Radiol 2008;68:214-26 CrossRef Medline

27. Lam SJ, Kumar S. Evolution of fetal ventricular dilatation in relation to severity at first presentation. J Clin Ultrasound 2014;42: 193-98 CrossRef Medline

28. Parazzini C, Righini A, Doneda C, et al. Is fetal magnetic resonance imaging indicated when ultrasound isolated mild ventriculomegaly is present in pregnancies with no risk factors? Prenat Diag 2012;32:752-57 CrossRef Medline

29. Limperopoulos C, Robertson RL, Estroff JA, et al. Diagnosis of inferior vermian hypoplasia by fetal magnetic resonance imaging: potential pitfalls and neurodevelopmental outcome. Am J Obstet Gynecol 2006;194:1070-76 CrossRef Medline

30. McQuillen PS, Barkovich A, Hamrick S, et al. Temporal and anatomic risk profile of brain injury with neonatal repair of congenital heart defects. Stroke 2007;38:736-41 CrossRef Medline

31. Petit CJ, Rome JJ, Wernovsky G, et al. Preoperative brain injury in transposition of the great arteries is associated with oxygenation and time to surgery, not balloon atrial septostomy. Circulation 2009; 119:709-16 CrossRef Medline

32. Glenn OA, Barkovich AJ. Magnetic resonance imaging of the fetal brain and spine: an increasingly important tool in prenatal diagnosis, part 1. AJNR Am J Neuroradiol 2006;27:1604-11 Medline

33. Chung R, Kasprian G, Brugger PC, et al. The current state and future of fetal imaging. Clin Perinatol 2009;36:685-99 CrossRef Medline

34. Friedman AH, Fahey JT. The transition from fetal to neonatal circulation: normal responses and implications for infants with heart disease. Semin Perinatal 1993;17:106-21 Medline

35. McKinstry RC, Miller JH, Snyder AZ, et al. A prospective, longitudinal diffusion tensor imaging study of brain injury in newborns. Neurology 2002;59:824-33 CrossRef Medline

36. Hartman RJ, Rasmussen SA, Botto LD, et al. The contribution of chromosomal abnormalities to congenital heart defects: a population-based study. Pediatr Cardiol 2011;32:1147-57 CrossRef Medline

37. Miller A, Riehle-Colarusso T, Alverson CJ, et al. Congenital heart defects and major structural noncardiac anomalies, Atlanta, Georgia, 1968 to 2005. J Pediatr 2011;159:70-78.e72 CrossRef Medline

38. Garel C, Chantrel E, Brisse H, et al. Fetal cerebral cortex: normal gestational landmarks identified using prenatal MR imaging. AJNR Am J Neuroradiol 2001;22:184-89 Medline 